\title{
Biomarkers of folate and related B-vitamins as predictors of cognitive decline in older Irish adults over a 5 year follow up period.
}

This abstract was awarded the student prize for best original communication presentation

Cognitive dysfunction and dementia are important public health issues in ageing, while optimal nutrition may be important in their prevention. One-carbon metabolism and the related B-vitamins may be important for maintaining cognitive health in ageing ${ }^{(1)}$ but few studies have investigated biomarker status of all relevant B-vitamins and furthermore studies in this area are typically observational cohort studies at one time point. The primary aim of this investigation was to examine the role of baseline status of folate and the metabolically related B-vitamins (vitamins B12, B6 and riboflavin) as predictors of subsequent cognitive decline over a five-year follow-up period in healthy older people.

From the total sample recruited to the Trinity, Ulster, Department of Agriculture (TUDA) Ageing Cohort Study ( $\geq 60$ years; $\mathrm{n}$ 5186), as previously described ${ }^{(2)}$, a sample of 2093 participants in Northern Ireland were potentially available, from which a sub-set (n 587) meeting the selection criteria (aged $>67$ years at baseline, MMSE score $>21$, no B12 injections) were recruited for reinvestigation 5 years after the initial TUDA study. The rate of cognitive decline was evaluated by re-assessment of cognition using the original battery of tests including the Repeatable Battery for the Assessment of Neuropsychological Status (RBANS), the Mini-Mental State Examination (MMSE) and the Frontal Assessment Battery (FAB). Accelerated cognitive decline was defined as the highest quartile of change in cognitive score over the 5 year follow up period; i.e. $\geq 7$ RBANS; $\geq 2$ MMSE; $\geq 3$ FAB points. At follow up, lower vitamin B6 status (PLP below median) and lower riboflavin status (EGRac above median) at baseline were each associated with an accelerated rate of cognitive decline, as measured by RBANS (Table). A similar relationship was shown with MMSE for vitamin B6 (OR: 1.485, CI: 1.002-2.200, p 0.049), but not for any other B-vitamin, while no significant relationships were observed for any B-vitamin biomarker with cognition as assessed by FAB.

\begin{tabular}{|c|c|c|c|}
\hline \multirow{2}{*}{ B-vitamin Biomarker status ${ }^{1}$} & \multicolumn{3}{|c|}{ Accelerated cognitive decline RBANS } \\
\hline & OR & $95 \% \mathrm{CI}$ & $\mathrm{p}$ value \\
\hline Plasma Homocysteine $(\mu \mathrm{mol} / \mathrm{L})$ Reference $<13.0$ vs $(13.0-27.5)$ & 0.967 & $0 \cdot 660-1.416$ & $0 \cdot 862$ \\
\hline RBC Folate (nmol/L) Reference $>868$ vs lower status $(185-865)$ & $1 \cdot 126$ & $0 \cdot 759-1 \cdot 670$ & $0 \cdot 556$ \\
\hline Serum Total B12 (pmol/L) Reference $>252$ vs lower status $(58-251)$ & $0 \cdot 828$ & $0 \cdot 564-1 \cdot 215$ & $0 \cdot 334$ \\
\hline Plasma PLP (B6) $(\mathrm{nmol} / \mathrm{L})$ Reference $>61 \cdot 3$ vs lower status $(11 \cdot 6-61 \cdot 3)$ & $1 \cdot 537$ & $1 \cdot 039-2 \cdot 273$ & $\mathbf{0 . 0 3 2}$ \\
\hline EGRac $(\mathrm{B} 2)^{*}$ Reference $<1.30$ vs lower status $(1.30-2.03)$ & 1.493 & $1 \cdot 013-2 \cdot 201$ & 0.043 \\
\hline
\end{tabular}

Binary logistic regression was performed with adjustment for age (years) and education (years)

Abbreviations: OR, odds ratio; CI, confidence interval; RBC, Red Blood Cell; PLP, Plasma Pyridoxal Phosphate; *EGRac, Erythrocyte Glutathione Reductase Activation Coefficient- higher EGRac ratio indicates lower riboflavin status.

${ }^{1}$ For each biomarker, participants with lower status were identified using the median value as the cut off point.

The results indicate that vitamin B6 and the metabolically related B-vitamin riboflavin, may have important roles in helping to maintain better cognitive health in ageing. Further research including RCTs targeted at those with lower B-vitamin status are warranted to investigate the role of all relevant B-vitamins in cognitive function.

1 Smith D, Refsum H (2016) Annual Review of Nutrition 36:211-39.

2 McCarroll K, Beirne A, Casey M et al. (2015) Age \& Ageing 44(5):847-853. 